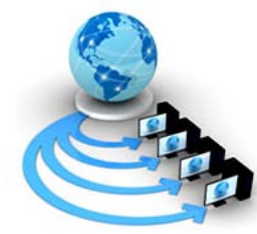

Volume 9, No. 2, March-April 2018

\title{
PROPOSE AND ENLARGEMENT OF PRIVACY PRESERVING TECHNIQUES IN BIG DATA MINING
}

\author{
P. Latha and \\ Research Scholar \\ Mewar University, Chittorgarh, Rajasthan, India
}

\author{
Dr. P. Niranjan \\ Research Supervisor \\ Mewar University, Chittorgarh, Rajasthan, India
}

\begin{abstract}
Big statistics describes huge information sets which have greater various and complex shapes like weblogs, social media, e-mail, sensors, and snapshots. This plenty less set up information and forte trends from traditional databases normally associated with greater headaches in storing, reading and applying similar strategies or extracting consequences. Big records analytics is the method of examining incredible portions of complex information on the manner to find out unseen patterns or spotting furtive correlations. Since conventional databases systems cannot be used to the method the massive information, it poses several demanding conditions to the studies community. Security and privacy are the crucial problems with facts. However, there exists incongruity some of the Big facts safety and privacy and the massive use of huge information. This paper offers insights on evaluating of huge facts, related worrying conditions, privateness and safety troubles and the differentiation among privateness \& protection requirements in huge records. Also, we cantered on numerous privacy fashions which may be stretched to massive statistics area, reading the blessings and downsides of Data anonymity privacy models.
\end{abstract}

Keywords: Security, Big data, privacy, database, inspecting.

\section{INTRODUCTION}

Due to present day technological improvement, the amount of information generated with the aid of social networking websites, sensor networks, Internet, healthcare applications, and masses of one in every of type organizations, is considerably growing every day. All the massive amount of records generated from particular sources in a couple of formats with very immoderate speed is referred as massive information. Big records have turn out to be a completely active research location for a final couple of years. The facts era price is developing so hastily that it is turning into distinctly difficult to deal with it the use of traditional techniques or structures[1]. Meanwhile, huge statistics can be based, semi-structured, or unstructured, which offers greater stressful conditions whilst appearing records storage and processing obligations. Therefore, to this give up, we need new approaches to save and examine data in real time. Big information, if captured and analyzed in a nicely timed manner, can be transformed into actionable insights which can be of the significant fee. It can assist groups and companies to improve the inner choice making energy and can create new opportunities thru facts evaluation. It also can assist to sell the scientific studies and monetary gadget thru remodeling conventional employer fashions and scientific values [2]. Despite large information may be successfully carried out for us to better apprehend the arena and innovate in numerous elements of human endeavors, the exploding quantity of statistics has stepped forward capacity privateness breach. For example, Amazon and Google can study our purchasing options and browsing behavior. Social networking websites together with Face book save all of the statistics about our private existence and social relationships.

\section{PREVIOUS STUDY}

This phase provides an evaluation of the literature on the safety of privacy of massive facts. Gheid and Challal proposed a privateness maintaining K-Means set of policies which protects privateness of statistics[3]. They used cryptography-loose multi-party additive scheme for horizontally portioned records. As cryptographic solutions degrade the regular performance of a system, it has grown to be essential to have opportunity solution[4]. As the information mining in allotted surroundings reasons privacy problems, their proposed solution plays clustering on big records with privateness preserved. Big facts refer to information this is streamed as well. Krempl et al. [5] examine on the trouble of protecting privateness of big information this is streaming. They found tough problems in mining such streaming records with recognizing to privacy protection of massive records. The disturbing situations embody streamed pre-processing, managing incomplete facts, dealing with skewed distributions, dealing with no longer on time facts, and desire of information dynamically. With respect to the lively mastering of records streams the annoying conditions recognized are uncertainty related to convergence, want for perpetual validation, temporal budget allocation, and performance bounds[6]. Other challenges associated with worrying situations of aggregation and worrying situations of studying. With understand to mining, there are worrying conditions associated with mining. They include incompleteness of records and locating houses of privacy retaining in modern datasets[7].

\section{METHODOLOGY}

Substitute touchy characteristic values with a few exclusive values. This decreases disclosure of private records. In a few situations, this clean substitution on my own wills, not cutting-edge anonymization techniques needs to be laboured. Occasional attributes in data necessities are 
substituted with well-known phrases. For instance, take into consideration some men and women who have a PG diploma are very a good deal less in worker database. A query which gathers person schooling qualifications and their deal with may be correlated and the end result set of a question which lists profits and age of the character can display the uniqueness of a candidate. This may be decreased via the usage of substituting qualification with every other often taking vicinity method called graduation which makes it complex to correlate.

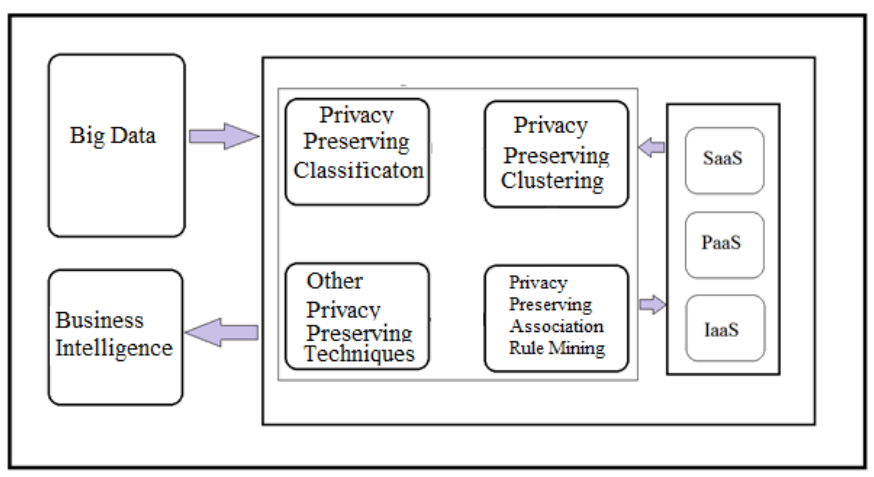

Fig.3.1. Proposed block diagram.

Security and Privacy in massive statistics is a vital trouble. Security focuses on defensive facts from pernicious attacks and stealing data for profits. Data privacy focuses at the use and governance of character's private records like making suggestions to ensure that customers' private statistics is being amassed, shared and completed in proper strategies. Although safety is important for shielding records however it's insufficient for addressing privateness.

\section{PRIVACY SECURITY}

Privacy Information privacy is the privilege to have a few control over how the private information is gathered and used. Information privateness is the potential of a character or organization to prevent information about them from turning into diagnosed to people other than those they deliver the information to. One excessive individual privacy trouble is the identification of personal data for the duration of transmission over the Internet. Security is the exercise of defensive statistics and records belongings thru the use of era, tactics, and schooling from-Unauthorized get proper of entry to, Disclosure, Disruption, Modification, Inspection, Recording, and Destruction. Privacy vs. Safety Data privateness is cantered at the use and governance of man or woman facts things like installing suggestions in location to make sure that clients' non-public information is being gathered, shared and utilized inappropriate methods. Security concentrates greater on protective facts from malicious attacks and the misuse of stolen data for profits. While protection is critical for protective facts, it's now not sufficient for addressing privateness.

\section{CONCLUSION}

Big information privateness is an important thing in these days' digital global wherein records is generated, accessed and shared notably with each species. It is now obligatory to promise privacy in huge information analytics. Privacy measures must now deliver emphasis at the uses of facts instead of statistics series. Techniques like statistics anonymization may be done to huge statistics however the problem lies within the fact that as duration and shape of information will increase, the probabilities of reidentification moreover boom. Thus, anonymization has a restricted potential within the subject of big information privacy. This paper offers a tremendous belief on large facts, privacy troubles and strategies.

\section{REFERENCES}

[1] D. Kornack and P. Rakic, "Cell Proliferation without Neurogenesis in Adult Primate Neocortex," Science, vol. 294, Dec. 2001,pp.2127-2130.

[2] L. Xu, C. Jiang, J. Wang, J. Yuan, and Y. Ren, "Information safety in large information: Privacy and information mining,” in IEEE Access, vol. 2, pp. 11491176, Oct. 2014.

[3] H. Hu, Y. Wen, T.-S. Chua, and X. Li, “toward scalable structures for huge information analytics: A generation educational," IEEE Access, vol. 2, pp. 652-687, Jul. 2014.

[4] Z. Xiao and Y. Xiao, "Security and privacy in cloud computing,'” IEEE Common. Surveys Tuts., vol. 15, no. 2, pp. 843-859, May 2013.

[5] C. Hongbing, R. Chunming, H. Kai, W. Weihong, and L. Yanyan, "Secure huge information garage and sharing scheme for cloud tenants," China Commun., vol. 12, no. 6, pp. 106-115, Jun. 2015.

[6] N. Cao, C. Wang, M. Li, K. Ren, and W. Lou, "Privacykeeping multi- key-word ranked search over encrypted cloud records,', IEEE Trans. Parallel Distrib. Syst., vol. 25, no. 1, pp. 222-233, Jan. 2014.

[7] O. M. Soundararajan, Y. Jenifer, S. Dhivya, and T. K. P. Rajagopal, "Data safety and privacy in cloud the usage of RC6 and SHA algorithms,'” Netw. Commun. Eng., vol. 6, no. Five, pp. 202-205, Jun. 2014. 